\title{
SONOGRAPHICALLY DIAGNOSED NON-ALCOHOLIC FATTY LIVER AS A PREDICTOR OF METABOLIC SYNDROME
}

\author{
Chandrajeet Yadav11, Sandeep Dhruw², Pulkit Shekhawatia 3 , Aman Gupta ${ }^{4}$ \\ ${ }^{1}$ Assistant Professor, Department of Radiodiagnosis, Sri Aurobindo Medical College, Indore, Madhya Pradesh. \\ ${ }^{2}$ Assistant Professor, Department of Radiodiagnosis, Sri Aurobindo Medical College, Indore, Madhya Pradesh. \\ 3 Post Graduate Resident, Department of Radiodiagnosis, Sri Aurobindo Medical College, Indore, Madhya Pradesh. \\ ${ }_{4}^{4}$ Professor and HOD, Department of Radiodiagnosis, Sri Aurobindo Medical College, Indore, Madhya Pradesh.
}

ABSTRACT
CONTEXT
Non-alcoholic fatty liver disease (NAFLD) is the most common liver disease, since its prevalence is estimated to be $20-30 \%$ in
general population of Western countries. Incidence of NAFLD in Indian population is on rise and its exact prevalence is not known. It
was thought to be a benign condition, but is now increasingly recognized as a major cause of liver-related morbidity and mortality.
Studies have proven that NAFLD may progress to cirrhosis, liver failure and hepatocellular carcinoma, in addition it has been shown
that NAFLD is strongly associated to the features of Metabolic Syndrome (MetS).

\section{AIMS AND OBJECTIVES}

To evaluate the association of sonographically diagnosed nonalcoholic fatty liver with metabolic syndrome and to estimate prevalence of nonalcoholic fatty liver in our population coming for general health check-up.

\section{METHOD}

We recruited 556 subjects, who visited for annual general health checkup at our institute. Based on exclusion criteria 148 subjects were excluded from the study and data from the remaining 408 subjects were included in the final statistical analysis. Characteristic ultrasound features such as increased hepatic echogenicity with attenuation of ultrasound beam and poor visibility of diaphragm and intrahepatic vessel borders were used to diagnose hepatic steatosis.

\section{RESULT}

Out of total 408 subjects who met with our inclusion criterias, 144 were diagnosed as having NAFLD (35.2\%) on sonography examination. Mean age of subjects in NAFLD group was 49.54 years; $79 \%$ of subjects in NAFLD group in our study had BMI $>25$, out of which $28.47 \%$ were morbidly obese. Tobacco use and lack of physical activity was observed to be more prevalent in NAFLD group. Out of total 144 subjects with NAFLD $43.75 \%$ were having metabolic syndrome. This association was statistically extremely significant ( $\mathrm{P}$ value $<0.0001$ ). Components of metabolic syndrome increased waist circumference, hypertension, impaired blood glucose level, low HDL cholesterol and high triglyceride level were more frequently observed in NAFLD group.

\section{CONCLUSION}

Issue whether or not NAFLD should be included as one of the components of the MetS is still in existence; however, it can be concluded that NAFLD is an important independent risk factor for prediction of MetS. The observations of our study might be helpful to support the clinical guideline and early intervention strategy for the NAFLD patients before being diagnosed as MetS.

\section{KEYWORDS}

Non-alcoholic fatty liver disease, Metabolic syndrome, Ultrasonography.

HOW TO CITE THIS ARTICLE: Yadav C, Dhruw S, Shekhawatia P, et al. Sonographically diagnosed non-alcoholic fatty liver as a predictor of metabolic syndrome. J. Evolution Med. Dent. Sci. 2016;5(15):722-726, DOI: 10.14260/jemds/2016/166

\section{INTRODUCTION}

Non-alcoholic fatty liver disease (NAFLD) is the most common liver disease, since its prevalence is estimated to be $20-30 \%$ in general population of Western countries.(1) Incidence of NAFLD in Indian population is on rise and its exact prevalence is not known in our part of world.

Financial or Other, Competing Interest: None.

Submission 09-01-2016, Peer Review 04-02-2016,

Acceptance 10-02-2016, Published 22-02-2016.

Corresponding Author:

Dr. Aman Gupta,

House No. 201, Dilpasand Avenue,

5/2, Manoramaganj, Indore-452001,

Madhya Pradesh.

E-mail:aman_sono@yahoo.co.in

DOI: $10.14260 /$ jemds $/ 2016 / 166$
The raising incidence of NAFLD in India may be attributed to recent lifestyle changes and growing prevalence of obesity. NAFLD occurs as a histological spectrum of disease and includes the subtypes of simple steatosis and Non-Alcoholic Steatohepatitis (NASH). It was thought to be a benign condition, but is now increasingly recognized as a major cause of liver-related morbidity and mortality.

Studies have proven that NAFLD may progress to cirrhosis, liver failure and hepatocellular carcinoma.(2) in addition it has been shown that NAFLD is strongly associated to the features of metabolic syndrome.(3-7)

Metabolic syndrome (MetS) has been defined by various national and international organizations. As per joint interim statement of international diabetes federation task force on epidemiology and prevention; National Heart, Lung and Blood 
Institute; American Heart Association; World Health Federation; International Atherosclerosis Society; and International Association for the Study of Obesity.(8) Presence of any three of the following cardiovascular risk factors is essential for metabolic syndrome:

1. Increased waist circumference males $>90 \mathrm{~cm}$ and females $>80 \mathrm{~cm}$.

2. Increased serum triglycerides $>150 \mathrm{mg} / \mathrm{dL}(1.7 \mathrm{mmol} / \mathrm{L})$.

3. Decreased serum HDL (Males $<40 \mathrm{mh} / \mathrm{dl}(1 \mathrm{mmol} / \mathrm{L})$ and females $<50 \mathrm{mg} / \mathrm{dL}(1.3 \mathrm{mmol} / \mathrm{L})$.

4. Increased blood pressure (Systolic blood pressure $>130 \mathrm{mmHg}$ and/or diastolic blood pressure $>85 \mathrm{mmHg}$ or taking antihypertensive treatment).

5. Increased blood sugar level (Fasting blood sugar $>100 \mathrm{mg} / \mathrm{dL} \quad(5.6 \mathrm{mmol} / \mathrm{L})$ or taking anti-diabetic treatment).

In general, NAFLD in the absence of NASH is an indolent disease with a benign course. However, as noted end-stage liver disease may occur as a consequence of NASH. The seriousness of this condition is demonstrated by the fact that approximately $50 \%$ of patients develop fibrosis, $15 \%$ develop cirrhosis and 3\% may advance to liver failure requiring transplantation. (9) The natural history of NAFLD is poorly understood and it is not known why some patients progress to cirrhosis while others do not. However, obesity and insulin resistance have been shown to be associated with more histological advanced disease. Long term outcome of NAFLD remains unclear and it may be a predictor of MetS, diabetes mellitus and ultimately the cardiovascular disease. A longterm followup is needed to conclude that morbidity and mortality among NAFLD patients is directed by cardiovascular risks or by liver related risks.

The aim of this observational study is to evaluate the association of sonographically diagnosed non-alcoholic fatty liver with metabolic syndrome and to estimate prevalence of non-alcoholic fatty liver in our population coming for general health check-up.

\section{MATERIAL AND METHODS}

\section{Study Population}

We recruited 556 subjects, who visited for annual general health checkup between January 2013 and June 2015 at our institute. Most of the subjects were executives in banks, post offices, other government organizations and companies. Morbidly obese patients attending general health check-up in our institute during the study period were also included. All the subjects who were alcoholic, having positive serologic marker for hepatitis B and C or other causes of chronic liver disease such as Wilson's disease, autoimmune hepatitis, etc. were excluded from the study. Based on these exclusion criteria, 148 subjects were excluded from the study. Data from the remaining 408 subjects were included in the final statistical analysis. It was a retrospective study in general health checkup subjects and written informed consent was not obtained from the subjects. No ethical issues were there in the study.

\section{STUDY DESIGN}

The health check-up programs were performed after an overnight fasting of at least 12 hours and all participants underwent a routine anthropometric, clinical and laboratory tests. The anthropometric measurements involved height, weight, blood pressure. Body Mass Index (BMI) was calculated and was used as an estimate of obesity. Blood pressure was measured in a sitting position. Blood samples were collected for biochemical analysis and values of cholesterol, triglycerides, LDL, HDL and fasting blood glucose were recorded. History of smoking, alcohol intake and physical activity was recorded from each subject.

All subjects were subjected to abdominal sonography, which is a part of general health check-up scheme in our region, ultrasound examination of abdomen was done on Philips iU22 ultrasound system using multi-frequency $1-4 \mathrm{MHz}$ convex transducer. B-Mode examination of each subject was done in supine and left lateral position to evaluate liver. Characteristic ultrasound features such as increased hepatic echogenicity as compared to renal cortical echogenicity with attenuation of ultrasound beam and poor visibility of diaphragm and intrahepatic vessel borders were used to diagnose hepatic steatosis (Fig. 1).

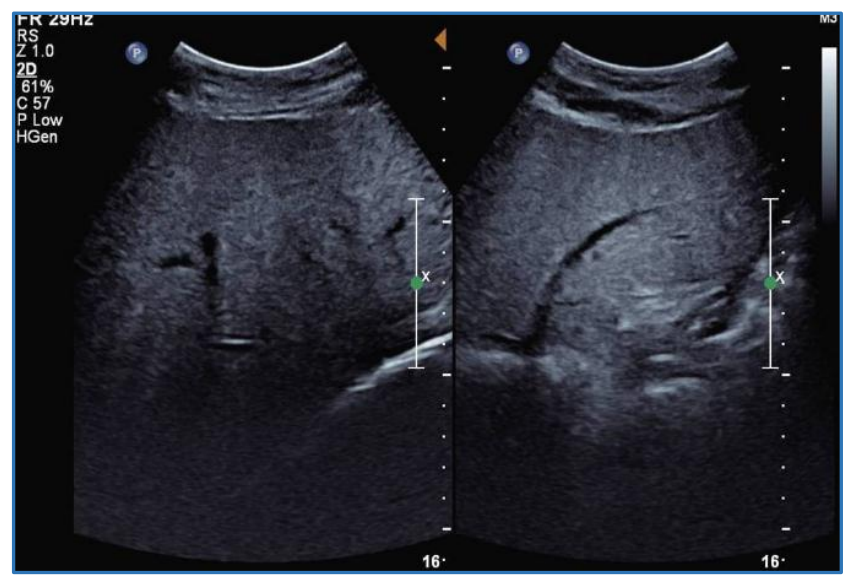

Fig. 1: USG image showing increased hepatic echogenicity with attenuation of ultrasound beam and poor visibility of diaphragm and intrahepatic vessel borders consistent with diagnosis of fatty liver

\section{STATISTICAL EVALUATION}

Metabolic syndrome was defined as per joint interim statement. Continuous variables are represented as Mean +/S.D. Categorical variables are presented as numbers with percentage. The statistically significant difference in continuous variables between subjects with and without NAFLD was calculated by unpaired t-test. Whereas statistically significant difference in categorically variables was evaluated by Chi square test. P value $<0.05$ was considered statistically significant.

\section{RESULTS}

Out of total 408 subjects who met with our inclusion criterias, 144 were diagnosed as having NAFLD (35.2\%) on sonography examination. Mean age of subjects in NAFLD group was 49.54 $(+/-6.6)$ years, whereas in non-NAFLD group $(n=264)$ mean age was $41.14(+/-9.65)$ years.

Majority of subjects (73.6\%) in NAFLD group were male, no significant sex discrimination was observed in non-NAFLD group. Following recommendation of WHO Asia-Pacific guideline, $28.47 \%$ of patients in NAFLD group were categorized as morbidly obese [BMI >40], 29.1\% obese [having BMI 30 to 40] and 31\% overweight [BMI-25 to 30]. In non- 
NAFLD group, $10.23 \%$ subjects were categorized as morbidly obese, $15.9 \%$ as obese, $28.4 \%$ as overweight and majority $45.45 \%$ as normal [BMI $<25]$. Mean waist circumference in NAFLD group was $105.9 \mathrm{cms}(+/-15.42)$, whereas in nonNAFLD group mean waist circumference was $93.27 \mathrm{cms}(+/-$ 19.18).

Tobacco use was observed to be more prevalent in NAFLD group (45.14\%), whereas in non-NAFLD group tobacco use was in $29.54 \%$. Lack of physical activity was more common in NAFLD group. A baseline characteristic of study population is presented in Table 1.

As per criterias laid down by joint interim statement of international diabetes federation task force on epidemiology and prevention; National Heart, Lung and Blood Institute; American Heart Association; World Health Federation; International Atherosclerosis Society and International Association for the Study of Obesity, 63 subjects (43.75\%) in NAFLD group with presence of three or more risk factors were labelled as of metabolic syndrome. In non-NAFLD group 7.2\% subjects were labelled as of MetS. Components of metabolic syndrome increased waist circumference, hypertension, impaired blood glucose level, low HDL cholesterol and high triglyceride level were more frequently observed in NAFLD group (Table 2).

\begin{tabular}{|c|c|c|c|c|c|}
\hline \multicolumn{2}{|c|}{ Variable } & Non-NAFLD & NAFLD & Total & P Value \\
\hline \multicolumn{2}{|c|}{ Sample Size } & 264 & 144 & 408 & \\
\hline \multicolumn{2}{|c|}{ Age (In years) Mean (+/- SD) } & $41.14(+/-9.65)$ & $49.54(+/-6.6)$ & $43.74(+/-9.5)$ & $<0.0001$ \\
\hline \multicolumn{2}{|c|}{ Gender } & & & & $<0.0001$ \\
\hline (a) & Male & $131(49.62 \%)$ & $106(73.61 \%)$ & 237 (58.09\%) & \\
\hline (b) & Female & $133(50.37 \%)$ & $38(26.39 \%)$ & $171(41.91 \%)$ & \\
\hline \multicolumn{6}{|c|}{ Body Mass Index } \\
\hline (a) & Normal & $120(45.45 \%)$ & $30(20.83 \%)$ & $150(36.76 \%)$ & $<0.0001$ \\
\hline (b) & Over weight & 75 (28.4\%) & $31(21.53 \%)$ & 106 (25.98\%) & 0.13 \\
\hline (c) & Obese & $42(15.9 \%)$ & $42(29.17 \%)$ & 84 (20.59\%) & 0.002 \\
\hline (d) & Morbidly obese & $27(10.23 \%)$ & $41(28.47 \%)$ & $68(16.67 \%)$ & $<0.0001$ \\
\hline \multicolumn{2}{|c|}{ Physical active } & $80(30.30 \%)$ & $16(11.1 \%)$ & $96(23.5 \%)$ & $<0.0001$ \\
\hline \multicolumn{2}{|c|}{ Tobacco use } & $78(29.54 \%)$ & $65(45.14 \%)$ & $143(35.05 \%)$ & 0.002 \\
\hline
\end{tabular}

Data are as means (Standard deviation) for continuous variable and frequency (Percentages) for categorical variables.

\begin{tabular}{|c|c|c|c|c|}
\hline Components of MetS & $\begin{array}{c}\text { Non- NAFLD } \\
(\mathbf{n = 2 6 4 )}\end{array}$ & $\begin{array}{c}\text { NAFLD } \\
(\mathbf{n = 1 4 4 )}\end{array}$ & $\begin{array}{c}\text { Total } \\
(\mathbf{n = 4 0 8})\end{array}$ & P Value \\
\hline $\begin{array}{c}\text { Waist Circumference in cms. } \\
\text { (Mean +/- SD) }\end{array}$ & $\begin{array}{c}93.27 \\
(+/-19.18)\end{array}$ & $\begin{array}{c}105.9 \\
(+/-15.42)\end{array}$ & $\begin{array}{c}97.1 \\
(+/-19.77)\end{array}$ & $<0.0001$ \\
\hline Hypertension & $57(21.6 \%)$ & $69(47.91 \%)$ & $126(30.9 \%)$ & $<0.0001$ \\
\hline Impaired Blood Glucose & $62(23.5 \%)$ & $56(38.9 \%)$ & $118(28.9 \%)$ & 0.001 \\
\hline Low HDL & $48(18.18 \%)$ & $52(36.11 \%)$ & $100(24.5 \%)$ & $<0.0001$ \\
\hline High Triglyceride & $34(12.88 \%)$ & $31(21.5 \%)$ & $65(15.9 \%)$ & 0.02 \\
\hline \multicolumn{2}{r}{} \\
\hline
\end{tabular}

Data are as means (Standard deviation) for continuous variable and frequency (Percentages) for categorical variables.

\section{DISCUSSION}

In this observational study carried out in subjects who turned up for annual general health checkup in our institute, prevalence of NAFLD on sonography examination was $35.2 \%$. Majority of these were males (73.6\%), the association being statistically extremely significant ( $\mathrm{P}$ value $<0.0001)$. Females constituted $26.4 \%$ of NAFLD group. Most of these females were postmenopausal. The difference in prevalence of NAFLD in male and female population has been attributed to protective effect of estrogen in premenopausal females. $(10,11)$

The incidence of NAFLD was seen increasing with increasing age of the subjects, mean age of subjects in NAFLD group was $49.54(+/-6.6)$, whereas in non-NAFLD group was $41.14(+/-9.65)$. This association was statistically extremely significant with $P$ value $<0.0001$. Increasing age as it brings restrictions on physical mobility may contribute to abdominal obesity and dyslipidemia. In another study NAFLD was associated with increased risk of cardiovascular disease, especially among older patients with elevated C-reactive protein levels.(12) A 79\% of subjects in NAFLD group in our study had BMI $>25$, out of which $28.47 \%$ were morbidly obese. Factors responsible for the development of NAFLD in obese patients are not clear. Insulin resistance is thought to be a primary pathophysiologic mechanism in development of fatty liver. Current understanding of the pathogenesis is as follows: Insulin resistance and visceral obesity lead to a hepatic influx of free fatty acids, resulting in increased triglyceride synthesis and decreased triglyceride export. This leads to hepatic steatosis.(13) Obesity is becoming an epidemic in Western as well as Eastern world and so the non-alcoholic fatty liver disease. $(14,15)$ In previous reports, authors have reported NAFLD prevalence around $57 \%-74 \%$ and $22 \%-58 \%$ of obese children and adults, respectively.(16) Incidence of obesity and 
non-alcoholic fatty liver disease is on rise in Indians as well, which is being observed in our study.

Out of total 144 subjects with NAFLD, 63 subjects $(43.75 \%)$ were having metabolic syndrome in our study. This association was statistically extremely significant (P value $<0.0001$ ). This strong association is consistent with other studies conducted on different sample sizes and different ethnic groups.(17-19) Metabolic syndrome is having higher incidence in Asian population.(20) which can be attributed to relative lack of exercises, central obesity and visceral fat deposition.(21) Hypertension, one of the cardiovascular risk factors essential for metabolic syndrome was observed in $47.91 \%$ of patients in NAFLD group in our study. In their study Hsiao et al.(18) demonstrated that presence of hepatic steatosis was significantly correlated with the prevalence and degree of hypertension. A study on non-obese and non-diabetic patients with primary hypertension has shown that hepatic steatosis prevalence can double as compared with control group.(22) Association of NAFLD and hypertension in our study was statistically extremely significant with $P$ value $<0.0001$.

Increased serum triglycerides and low HDL was observed in $21.5 \%$ and $36.1 \%$ of subjects in NAFLD group respectively. Cotrim et al.(23) reported the presence of hyperlipidemia in $66.8 \%$ of patients with NAFLD in Brazil. Association of high serum triglyceride with NAFLD in our study was statistically significant $(P$ value $=0.02$ ), whereas low HDL showed extremely significant association ( $P$ value $<0.0001$ ). Marchesini G, et al.(4) reported hypertriglyceridemia and low HDL-cholesterol level in $64 \%$ and $30-42 \%$ of NAFLD patients respectively.

Impaired blood glucose was observed in $38.9 \%$ of subjects in NAFLD group in our study. The association was statistically significant with $P$ value 0.001 . In Brazil, the prevalence of metabolic syndrome and diabetes among patients with NAFLD was estimated at $41.3 \%$ and $22.7 \%$, respectively.(23) The association of both conditions is related with more aggressive disease and increasing mortality.(24)

The association between NAFLD and MetS could be mainly explained by insulin resistance, which was the main mechanism linking NAFLD and MetS from pathophysiological perspective. $(25,26)$ Many researches demonstrated that insulin resistance was a pathogenic factor of the metabolic syndrome and also played a major role in the development and progression of NAFLD.(27) In NAFLD population, excess fat accumulation in the hepatic parenchyma or overabundance of fatty acids was a major contributor to the development of insulin resistance and subsequent MetS.(28,29)

\section{CONCLUSION}

Issue whether or not NAFLD should be included as one of the components of the MetS is still in existence; however, it can be concluded that NAFLD is an important independent risk factor for prediction of MetS. The observations of our study might be helpful to support the clinical guideline and early intervention strategy for the NAFLD patients before being diagnosed as MetS. Therefore, as for the NAFLD detected in the health checkup programmes could help initiate weight and dietary control, so as to prevent the development of MetS and even the cardiovascular diseases.

Some limitations in our study needs to be highlighted. First, NAFLD in our study was detected on basis of grey scale sonographic features alone higher imaging modalities, such as shear-wave elastography, MRI were not soughted out and pathological correlation was not done. Secondly, our study data is based on the subjects reporting for routine health check-up system in urban population of Indore district, further longitudinal and cross-sectional studies with large sample volumes need to be done to confirm the value of NAFLD as a marker for predicting the risk of metabolic syndrome.

\section{REFERENCES}

1. Bedogni G, Miglioli L, Masutti F, et al. Prevalence of and risk factors for non-alcoholic fatty liver disease: the Dionysos nutrition and liver study. Hepatology 2005;42:44-52

2. Adams LA, Lymp JF, St Sauver J. The natural history of nonalcoholic fatty liver disease: a population-based cohort study. Gastroenterology 2005;129:113-21.

3. Kwon YM, Oh SW, Hwang SS, et al. (2012). Association of non-alcoholic fatty liver disease with components of metabolic syndrome according to body mass index in Korean adults. Am J Gastroenterol 2012;107:1852-58.

4. Marchesini G, Bugianesi E, Forlani G, et al. Non-alcoholic fatty liver, steatohepatitis and the metabolic syndrome. Hepatology 2003;37:917-23.

5. Targher G, Day CP, Bonora E (2010). Risk of cardiovascular disease in patients with non-alcoholic fatty liver disease. $\mathrm{N}$ Engl J Med Overseas Ed 363:1341-50.

6. Marchesini G, Brizi M, Bianchi G, et al. (2001). Nonalcoholic fatty liver disease: a feature of the metabolic syndrome. Diabetes 50:1844-50.

7. Smits MM, Ioannou GN, Boyko EJ, et al. (2013). Nonalcoholic fatty liver disease as an independent manifestation of the metabolic syndrome: results of an US national survey in three ethnic groups. J Gastroenterol Hepatol 28:664-70.

8. Alberti KG, Eckel RH, Grundy SM, et al. Harmonizing the metabolic syndrome. Circulation 2009;120:1640-5.

9. Sheth SG, Gordon FD, Chopra S. Non-alcoholic steatohepatitis. Ann Intern Med 1997;126:137-145.

10. Ibrahim MM. Subcutaneous and visceral adipose tissue: structural and functional differences. Obes Rev 2010;11:11-18.

11. Ayonrinde OT, Olynyk JK, Beilin LJ, et al. Gender-specific differences in adipose distribution and adipocytokines influence adolescent non-alcoholic fatty liver disease. Hepatology 2011;53:800-809.

12. Chiang $\mathrm{CH}$, Huang $\mathrm{CC}$, Chan $\mathrm{WL}$, et al. The severity of nonalcoholic fatty liver disease correlates with high sensitivity C-reactive protein value and is independently associated with increased cardiovascular risk in healthy population. Clin Biochem 2010;43:1399-404.

13. Gill HK, Wu GY. Non-alcoholic fatty liver disease and the metabolic syndrome: effects of weight loss and a review of popular diets. Are low carbohydrate diets the answer? World J Gastroenterol 2006;12(3):345-53.

14. Wong VW. Non-alcoholic fatty liver disease in Asia: a story of growth. J Gastroenterol Hepatol 2013;28:18-23.

15. Younossi ZM, Stepanova M, Afendy M, et al. Changes in the prevalence of the most common causes of chronic liver diseases in the United States from 1988 to 2008. Clin Gastroenterol Hepatol 2011;9:524-30.

16. Tarantino G, Saldalamacchia G, Conca P, et al. Non-alcoholic fatty liver disease: further expression of the metabolic syndrome. J Gastroenterol Hepatol 2007;22:293-3. 
17. Chen SH, He F, Zhou HL, et al. Relationship between nonalcoholic fatty liver disease and metabolic syndrome. J Dig Dis 2011;12:125-30.

18. Hsiao PJ, Kuo KK, Shin SJ, et al. Significant correlations between severe fatty liver and risk factors for metabolic syndrome. J Gastroenterol Hepatol 2007;22:2118-23.

19. Marchesini G, Brizi M, Bianchi G. Non-alcoholic fatty liver disease: a feature of the metabolic syndrome. Diabetes 2001;50:1844-50.

20. Wong RJ, Ahmed A. Obesity and non-alcoholic fatty liver disease: disparate associations among Asian populations. World J Hepatol 2014;6:263-73.

21. Koehler EM, Schouten JN, Hansen BE, et al. Prevalence and risk factors of non-alcoholic fatty liver disease in the elderly: results from the Rotterdam study. J Hepatol 2012;57:1305-11.

22. Donati G, Stagni B, Piscaglia F, et al. Increased prevalence of fatty liver in arterial hypertensive patients with normal liver enzymes: role of insulin resistance. Gut 2004;53:1020-23.
23. Cotrim HP, Parise ER, Oliveira CP, et al. Non-alcoholic fatty liver disease in Brazil. Clinical and histological profile. Ann Hepatol 2011;10:33-37.

24. Younossi ZM, Gramlich T, Matteoni CA, et al. Non-alcoholic fatty liver disease in patients with type 2 diabetes. Clin Gastroenterol Hepatol 2004;2:262-5.

25. Angelico F, Del BM, Conti R, et al. Insulin resistance, the metabolic syndrome and non-alcoholic fatty liver disease. J Clin Endocrinol Metab 2005;90:1578-82.

26. Angulo P. Non-alcoholic fatty liver disease. N Engl J Med 2002 Overseas Ed; 346:1221-31.

27. Tilg H, Moschen AR. Insulin resistance, inflammation and non-alcoholic fatty liver disease. Trends in endocrinology and metabolism 2008;19:371-79.

28. Bhatia LS, Curzen NP, Calder PC, et al. Non-alcoholic fatty liver disease: a new and important cardiovascular risk factor. Eur Heart J 2012;33:1190-200.

29. Bugianesi E, McCullough AJ, Marchesini G. Insulin resistance: a metabolic pathway to chronic liver disease. Hepatology 2005;42:987-1000. 